\title{
Avaliação da gestão de qualidade em Unidade de Alimentação e Nutrição: estudo de viabilidade
}

\author{
Evaluation of the quality Management in the Food and Nutrition Unit: feasibility study \\ Evaluación de la gestión de la calidad en la Unidad de Alimentación y Nutrición: estudio de \\ viabilidade
}

Recebido: 06/10/2021 | Revisado: 13/10/2021 | Aceito: 14/10/2021 | Publicado: 16/10/2021

\author{
Joeli Silva de Souza \\ ORCID: https://orcid.org/0000-0002-2336-3555 \\ Universidade Federal da Bahia, Brasil \\ E-mail: joeli.souza@ufba.br \\ Natália Andrade dos Santos \\ ORCID: https://orcid.org/0000-0002-0830-7387 \\ Universidade Federal da Bahia, Brasil \\ E-mail: nataliaandrade.nut@gmail.com \\ Carlos Rodrigo Nascimento de Lira \\ ORCID: https://orcid.org/0000-0001-7266-1367 \\ Universidade Federal da Bahia, Brasil \\ E-mail: carlos.rodrigo.n@hotmail.com \\ Rafaela dos Santos Bomfim \\ ORCID: https://orcid.org/0000-0001-5908-9597 \\ Universidade Federal da Bahia, Brasil \\ E-mail: rafaelasantos9116@ hotmail.com \\ Maria da Conceição Pereira da Fonseca \\ ORCID: https://orcid.org/0000-0003-0325-7162 \\ Universidade Federal da Bahia, Brasil \\ E-mail: mcfonseca@gmail.com
}

\begin{abstract}
Resumo
O estudo teve como objetivo avaliar as condições higienicossanitárias e estruturais de uma Unidade de Alimentação e Nutrição, propondo o desenvolvimento de estratégias para a implantação de programas de gestão de qualidade. Estudo de caráter exploratório, com abordagem qualitativa e quantitativa dos dados, sendo abordado um serviço de alimentação e nutrição. Para a avaliação das condições higienicossanitárias foram utilizados check list diário e semanal. Em relação aos resultados, baixas pontuações gerais e altas frequências de condições insatisfatórias foram observadas em parte dos setores avaliados. Identificaram-se os setores de panelas e pré-preparo de saladas com maiores itens de não conformidade e o setor de higienização de bandejas e utensílios com maior pontuação em relação às conformidades. Diante dos resultados encontrados, percebe-se que a Unidade de Alimentação e Nutrição apresentou condições higienicossanitárias desfavoráveis, fato este que pode comprometer a segurança dos alimentos preparados nestes locais. O seguimento de boas práticas e o cumprimento do mesmo pela Unidade de Alimentação e Nutrição é indispensável para a produção de alimentos seguros que contribuam com a saúde e bem estar dos comensais.

Palavras-chave: Lista de verificação; Serviços de alimentação; Condições higienicossanitárias; Controle de qualidade.

Abstract

The study aimed to evaluate the hygienic-sanitary and structural conditions of a Food and Nutrition Unit, proposing the development of strategies for the implementation of quality management programs. Exploratory study, with a qualitative and quantitative approach to data, addressing a food and nutrition service. For the assessment of hygienicsanitary conditions, daily and weekly checklists were used. Regarding the results, low overall scores and high frequencies of unsatisfactory conditions were observed in part of the evaluated sectors. The sectors of pans and prepreparation of salads with the highest non-compliance items and the cleaning of trays and utensils sector with the highest score in terms of compliance were identified. In view of the results found, it is clear that the Food and Nutrition Unit presented unfavorable hygienic-sanitary conditions, a fact that can compromise the safety of food prepared in these places. The follow-up of good practices and compliance with them by the Food and Nutrition Unit is essential for the production of safe foods that contribute to the health and well-being of diners.
\end{abstract}

Keywords: Check list; Food services; Hygienic and sanitary conditions; Quality control. 


\section{Resumen}

El estudio tuvo como objetivo evaluar las condiciones higiénico-sanitarias y estructurales de una Unidad de Alimentación y Nutrición, proponiendo el desarrollo de estrategias para la implementación de programas de gestión de la calidad. Estudio exploratorio, con enfoque cualitativo y cuantitativo de datos, abordando un servicio de alimentación y nutrición. Para la evaluación de las condiciones higiénico-sanitarias se utilizaron listas de verificación diarias y semanales. En cuanto a los resultados, se observaron puntuaciones generales bajas y altas frecuencias de condiciones insatisfactorias en parte de los sectores evaluados. Se identificaron los sectores de sartenes y prepreparación de ensaladas con mayor incumplimiento de los ítems y el sector de limpieza de bandejas y utensilios con mayor puntaje en términos de cumplimiento. A la vista de los resultados encontrados, es evidente que la Unidad de Alimentación y Nutrición presentó condiciones higiénico-sanitarias desfavorables, hecho que puede comprometer la inocuidad de los alimentos preparados en estos lugares. El seguimiento de las buenas prácticas y el cumplimiento de las mismas por parte de la Unidad de Alimentación y Nutrición es fundamental para la producción de alimentos seguros que contribuyan a la salud y bienestar de los comensales.

Palabras clave: Lista de verificación; Servicios de comida; Condiciones higiénicas y sanitarias; Control de calidad.

\section{Introdução}

O sucesso das organizações depende da qualidade de sua administração, pois são os administradores quem estabelecem objetivos e guiam a organização de forma a alcançá-las, propondo mudanças e adaptando-as a um ambiente cada vez mais dinâmico. A administração foi definida por Mary Parket Follet como a arte de produzir bens ou serviços por intermédio das pessoas, é um processo na medida em que consiste em um conjunto de atividades e tarefas relacionadas a fim de atingir um objetivo comum (Sobral \& Peci, 2008). O papel do administrador em épocas de mudanças e instabilidades centra-se, sobretudo na inovação do que na manutenção do status organizacional (Chiavenato, 2003).

As Unidades de Alimentação e Nutrição - UAN pertencem ao setor de alimentação coletiva, cuja finalidade é desempenhar atividades relacionadas à alimentação e nutrição, ao mesmo tempo em que mantém padrões higienicosanitários no armazenamento de alimentos, produção e distribuição para o consumo. O controle da higiene e das condições sanitárias onde os alimentos são preparados é um fator crucial para a melhoria da qualidade dos produtos e serviços (Susin, Pereira, Gregoletto \& Cremonese, 2017). O processo administrativo e seus componentes têm influência direta com a obtenção de qualidade em uma UAN, uma vez que as doenças veiculadas por alimentos ou de origem alimentar se propagam rapidamente e com alta patogenicidade. O nutricionista, portanto, está apto para a gestão destas Unidades, organizando, planejando, coordenando e avaliando os aspectos administrativos de forma a aperfeiçoar e conferir qualidade sanitária e nutricional ao serviço.

A gestão da qualidade facilita o processo de mudanças organizacionais envolvendo planejamento e controle, criando processos que facilitem a melhora de todos os aspectos de desempenho da linha de produção. O produto com garantia e qualidade traduz a fidelidade aos padrões convencionados e revelam o conhecimento e a observância criteriosa às etapas e rotinas do processo produtivo e o detalhamento de como produzir. Como resultado, as empresas têm implementado os chamados Sistemas de Gestão de Qualidade - SGQ, que tem como finalidade a aplicação de métodos e mecanismos de garantia na qualidade da produção de alimentos e processos com padrões de qualidade pré-estabelecidos como ferramentas de segurança alimentar (Sousa, 2019).

Para garantia da qualidade dos alimentos produzidos nos serviços de alimentação e nutrição, são utilizados vários instrumentos de gestão da qualidade, entre eles destacam-se: a adoção de Boas Práticas de Fabricação - BPF dos alimentos, o conhecimento e adoção dos Padrões e Procedimentos Operacionais de Sanitização - PPOS/SSOP, bem como a aplicação do sistema de Análise de Perigos e Pontos Críticos de Controle- APPCC (Abreu, Spinelli \& Zanardi, 2007). Em relação ao serviço de alimentação e nutrição, a qualidade está associada a aspectos intrínsecos do alimento sendo a qualidade sensorial e nutricional do alimento, à segurança que está relacionada às qualidades higienicossanitárias, ao atendimento no que se refere à relação cliente-fornecedor e ao preço (Olmedo et al., 2017). 
De acordo com a Agência Nacional de Vigilância Sanitária - ANVISA, dentre as legislações vigentes com maior aplicabilidade aos estabelecimentos fornecedores de alimentação coletiva que visam às boas práticas de fabricação destacamse: as Portarias $n^{\circ} 1.428$ de 26/12/1993 (Brasil, 1993) e 326 de 30/07/1997 (Brasil, 1997) que constituem as orientações necessárias para inspeção sanitária por meio da verificação do Sistema APPCC da empresa produtora e de serviços de alimentos e os aspectos que devem ser levados em conta para a aplicação de BPF, respectivamente. A Resolução da Diretoria Colegiada - RDC no 275, de 21/10/2002 (Brasil, 2002) que aprova o Regulamento Técnico de Procedimentos Operacionais Padronizados - POP e a Lista de verificação das Boas Práticas de Fabricação e a Portaria nº 216, de 15/09/2004 (Brasil, 2004), que dispõe sobre o Regulamento Técnico de Boas Práticas para Serviços de Alimentação.

Uma das ferramentas mais utilizadas para adequação das Boas Práticas é a lista de verificação, também chamado de check list para a área de alimentos (Stedefeldt et al., 2013; Almeida, Amor \& Silva, 2018). Esta nos permite realizar avaliação preliminar das condições higienicossanitárias de um estabelecimento produtor de alimentos. Os requisitos avaliados são relativos a recursos humanos; condições ambientais; instalações; edificações e saneamento; equipamentos; sanitização; produção; embalagem e rotulagem; controle de qualidade; e controle no mercado. Esta avaliação inicial possibilita diagnosticar pontos críticos, e a partir dos dados delinearem ações corretivas para adequação dos setores críticos, buscando minimizar riscos físicos, químicos e biológicos que comprometam a saúde do comensal (Sousa, 2017; Quintino \& Rodolpho, 2018).

Com base no descrito, esta avaliação se faz necessária, pela importância de avaliar criticamente as possibilidades de implantação de ferramentas de gestão de qualidade, que são importantes recursos para a obtenção da garantia da qualidade, para que possa ser utilizado para estabelecer ações corretivas visando à melhora das condições higienicossanitárias, enfatizando a importância dos programas como: BPF, APPCC e POP.

Diante do exposto, o objetivo deste trabalho foi avaliar as condições higienicossanitárias e estruturais de uma Unidade de Alimentação e Nutrição, propondo o desenvolvimento de estratégias para a implantação de programas de gestão da qualidade.

\section{Metodologia}

Trata-se de um estudo quantitativo e qualitativo, de caráter observacional e de diagnóstico, tendo como objeto de estudo um Serviço de Alimentação e Nutrição na modalidade de Restaurante Universitário - RU, em Salvador - BA, realizado no período de janeiro de 2017.

Os serviços de alimentação universitários são voltados para a produção e fornecimento de refeições principalmente para comensais universitários, que atualmente é executado pelo Programa Nacional de Assistência Estudantil - PNAES, que além de auxiliar a alimentação, também oferece assistência à moradia estudantil, à saúde, inclusão digital, cultura, esporte, creche e apoio pedagógico. As refeições oferecidas nos RU funcionam como importante suporte alimentar para os estudantes, contribuindo desta forma com sua saúde e no desenvolvimento mental e físico, logo, é necessário atentar-se à qualidade dessas refeições (Brasil, 2010).

A UAN em estudo adotava a modalidade de gestão por terceiros, tendo como base o termo de referência estabelecido entre uma empresa terceirizada e a Universidade. A produção de refeições estava distribuída entre almoço (das 11h00min às 14h00min), jantar (das $17 \mathrm{~h} 00 \mathrm{~min}$ às $20 \mathrm{~h} 00 \mathrm{~min}$ ) e refeições transportadas, com cerca de 2.800 refeições por dia. A unidade contava com uma média de 55 funcionários em seu quadro total. O RU funcionava todos os dias do ano, com exceção do período de carnaval, pois a Universidade fechava por localizar-se em um dos circuitos da festa. Nos finais de semana o funcionamento acontecia com um quantitativo reduzido de estudantes devido não haver aula aos sábados para a maioria dos cursos, sendo que os bolsistas eram o público mais frequente nesses dois dias. $\mathrm{O}$ atendimento era do tipo self-service para as duas opções de saladas e porcionado para o prato principal, opção ovolactovegetariana, acompanhamento, guarnição, 
sobremesa e bebida não alcoólicos. Contava como responsável técnica, no caso, uma nutricionista da empresa, e a mesma era supervisionada pelo Núcleo de Segurança Alimentar da Universidade.

Como primeira etapa para a avaliação do serviço de alimentação, foram utilizadas duas listas de verificação (uma para inspeção diária e outra para inspeção semanal), sendo este método, o mais utilizado em território brasileiro. Essa etapa visou avaliar as condições higienicossanitárias bem como as não conformidades. Devido à falta de um instrumento padronizado para este fim, as listas de verificação utilizadas foram adaptadas para contemplarem todos os itens e critérios de avaliação (Stedefeldt et al., 2013). A adaptação levou em consideração principalmente as bases do termo de referência entre a concessionária e a Universidade que estava em vigência no período do estudo.

Todos os setores da unidade foram avaliados durante uma semana a partir de visitas de inspeção, analisando, portanto, as condições sanitárias das instalações, condições de armazenamento dos alimentos, produção das refeições, higiene do ambiente e dos manipuladores de alimentos. A lista de verificação para inspeção diária foi utilizada para avaliação do controle sanitário na produção das refeições, enquanto que a lista de verificação para inspeção semanal referia-se à identificação do serviço e caracterização das condições de higiene da estrutura física da unidade. Cada lista de verificação tinha perguntas específicas por setores da unidade, o aspecto a ser observado e a quantidade de itens em cada um (Quadro 1). O preenchimento dos itens avaliados ocorria mediante as observações em cada setor, sendo, portanto, um método rápido, de baixo custo, prático e de grande benefício, pois permitiu estabelecer as proposições de mudanças nos setores, como também possibilidade de implantação de programas de gestão de qualidade, a partir das necessidades do serviço. 
Quadro 1. Aspectos avaliados por setores com uso das listas de verificação (semanal e diária). Salvador, Bahia, 2017.

\begin{tabular}{|c|c|c|}
\hline \multicolumn{3}{|c|}{ LISTA DE VERIFICAÇÃO SEMANAL } \\
\hline Setores & Aspectos avaliados & $\mathbf{N}^{\circ}$ de itens \\
\hline Cocção & Estrutura das paredes, teto, portas e janelas. & 04 \\
\hline Açougue & Estrutura das paredes, teto, portas e janelas. & 04 \\
\hline Pré-preparo de saladas & Estrutura das paredes, teto, portas e janelas. & 04 \\
\hline Sobremesa & Estrutura das paredes, teto, portas e janelas. & 04 \\
\hline Higienização de panelas & Estrutura das paredes, teto, portas e janelas. & 04 \\
\hline Higienização de bandejas e utensílios & Estrutura das paredes, teto, portas e janelas. & 04 \\
\hline Recepção de gêneros alimentícios & $\begin{array}{l}\text { Qualificação de fornecedor, recepção e avaliação de } \\
\text { mercadorias. }\end{array}$ & 16 \\
\hline Estoque refrigerado: Antecâmara & Organização e limpeza do setor. & 08 \\
\hline $\begin{array}{l}\text { Estoque refrigerado: Câmara de } \\
\text { hortifrutigranjeiro }\end{array}$ & $\begin{array}{l}\text { Limpeza do setor, risco de contaminação física e } \\
\text { química, contaminação cruzada, controle de } \\
\text { temperatura e higienização de hortifrúti. }\end{array}$ & 21 \\
\hline Estoque refrigerado: Câmara de refrigerados & $\begin{array}{l}\text { Limpeza do setor, risco de contaminação física e } \\
\text { química, contaminação cruzada e controle de } \\
\text { temperatura. }\end{array}$ & 18 \\
\hline Estoque refrigerado: Câmara de congelados & $\begin{array}{l}\text { Limpeza do setor, risco de contaminação física e } \\
\text { química, contaminação cruzada e controle de } \\
\text { temperatura. }\end{array}$ & 16 \\
\hline Estoque seco & $\begin{array}{l}\text { Avaliação de setor, identificação, controle de } \\
\text { validade, aspectos gerais de cuidados no } \\
\text { armazenamento. }\end{array}$ & 20 \\
\hline Estoque de descartáveis & Condições gerais e material utilizado. & 14 \\
\hline Sala da Nutrição & Limpeza e organização. & 12 \\
\hline \multicolumn{2}{|c|}{ Total de itens avaliados } & 149 \\
\hline \multicolumn{3}{|c|}{ LISTA DE VERIFICAÇÃO DIÁRIA } \\
\hline Setores & Aspectos avaliados & $\mathbf{N}^{\circ}$ de itens \\
\hline Recepção de gêneros alimentícios & $\begin{array}{l}\text { Condições gerais de limpeza, qualificação de } \\
\text { fornecedor, recepção e avaliação de mercadorias. }\end{array}$ & 17 \\
\hline Cocção & $\begin{array}{l}\text { Controle de temperaturas, condições gerais de } \\
\text { limpeza e higiene pessoal, hábitos higiênicos, } \\
\text { higienização das mãos dos manipuladores. }\end{array}$ & 28 \\
\hline Açougue & $\begin{array}{c}\text { Limpeza do setor, risco de contaminação física e } \\
\text { química, contaminação cruzada, higiene pessoal, } \\
\text { hábitos higiênicos, higienização das mãos dos } \\
\text { manipuladores. }\end{array}$ & 26 \\
\hline Pré-preparo de saladas & $\begin{array}{c}\text { Limpeza do setor, risco de contaminação física e } \\
\text { química, contaminação cruzada, higiene pessoal, } \\
\text { hábitos higiênicos, higienização das mãos dos } \\
\text { manipuladores. }\end{array}$ & 34 \\
\hline Sobremesas & $\begin{array}{c}\text { Limpeza do setor, risco de contaminação física e } \\
\text { química, contaminação cruzada, higiene pessoal, } \\
\text { hábitos higiênicos, higienização das mãos dos } \\
\text { manipuladores. }\end{array}$ & 29 \\
\hline Higienização de panelas & $\begin{array}{l}\text { Aspectos gerais de equipamentos e condições gerais } \\
\text { de limpeza. }\end{array}$ & 18 \\
\hline Higienização de bandejas e utensílios & $\begin{array}{l}\text { Aspectos gerais de equipamentos e condições gerais } \\
\text { de limpeza. }\end{array}$ & 23 \\
\hline Distribuição & Temperatura e tempo de exposição do alimento. & 14 \\
\hline Refeitório & Condições gerais de limpeza. & 8 \\
\hline Catação de grãos e preparo de sucos & Condições gerais de limpeza e contaminação cruzada. & 10 \\
\hline \multicolumn{2}{|c|}{ Total de itens avaliados } & 207 \\
\hline
\end{tabular}

Fonte: Dados da pesquisa (2017).

A partir dos itens observados, cada afirmativa da lista de verificação tinha como opções de resposta: SIM, quando o aspecto avaliado estivesse de acordo à legislação; NÃO, quando não estivesse de acordo ao recomendado; e NSA quando não 
se aplicasse a realidade do local e considerou-se pontuação um para todos os itens. Para o cálculo da pontuação final foi utilizada a fórmula utilizada por Cardoso et al. (2010), onde:

$$
\text { Escore Obtido }(E O)=\frac{\Sigma \text { dos pontos positivos } \times 100}{\Sigma \text { dos pontos possiveis }-\Sigma \text { dos pontos dos itens não aplicáveis }}
$$

Sendo: $\Sigma($ soma $)$ dos pontos possíveis $=$ todos os itens $\mathrm{SIM}+\mathrm{NÃO}+\mathrm{NSA}$.

Assim, com base na frequência da pontuação, a unidade foi classificada em: $<30 \%$ (critico); $\geq 30 \%$ e $<50 \%$ (insatisfatório); $\geq 50 \%$ e $<70 \%$ (regular); $\geq 70 \%$ e $<90 \%$ (bom); $\geq 90 \%$ (excelente). Além disso, para a avaliação das condições higienicossanitárias foi adotado como embasamento as bases contratuais vigentes entre a Universidade e a empresa terceirizada. Assim, com a utilização da lista de verificação foi possível propor mudanças nos setores, como também propor a possibilidade de implantação de programas de gestão de qualidade, a partir das necessidades do serviço. Este estudo faz parte de um projeto maior, aprovado pelo Comitê de Ética da Escola de Nutrição da Universidade Federal da Bahia (Parecer $n^{\circ}$ 228.318/2012).

\section{Resultados e Discussão}

Com aplicação da lista de verificação diária na Unidade, um escore global de $81,41 \%$ foi obtido, sugerindo, portanto, que a UAN estava em boa classificação com relação às condições higienicossanitárias. Entretanto, ao avaliar a Unidade por setores separadamente, é perceptível que muitos deles apresentaram-se com qualidade regular, a exemplo dos setores de cocção, açougue, pré-preparo de salada, sobremesa e higienização de panelas, pois obtiveram escore abaixo de 70\% (Tabela 1).

Estes achados podem ter sofrido influência do horário de aplicação do instrumento, que dependendo do horário em que aplicou-se, o processo produtivo de refeições poderia está em pleno andamento e para tal, alguns itens da lista de verificação não puderam obter resposta sim, como por exemplo, a organização na área de cocção, conversa entre os manipuladores no açougue, fluxo de produção inadequado nos setores de pré-preparo de salada, sobremesa e higienização de panelas. Outro fator que pode ter corroborado para os achados foi a falta de padronização do processo de trabalho. Os achados deste estudo assemelham-se aos de Ribeiro et al. (2018), que avaliaram as Unidades de Alimentação de seis escolas municipais em São Paulo, apresentando uma média de pontuação de 48,51\%, estando assim categorizadas como "risco sanitário alto".

Desta forma, sugere-se que maior atenção, treinamento e educação devem ser direcionados aos manipuladores de alimentos, os quais possuem parte da responsabilidade pela produção, higiene e segurança das refeições produzidas, desta forma, o processamento e serviços são limites cruciais para prevenção da maioria das doenças veiculadas por alimentos. Em estudo com 172 manipuladores de alimentos no Espírito Santo - Brasil, e em outro estudo com 70 manipuladores de restaurante populares no Brasil, foram evidenciados que os treinamentos contínuos em segurança alimentar favorecem o conhecimento, atitudes e práticas de segurança alimentar desses manipuladores (Souza, Azevedo \& Seabra, 2018; Vitória, et al., 2021).

As ferramentas da qualidade são importantes recursos para o alcance e garantia da qualidade e da gestão estratégica, que podem ser aprimoradas através do desenvolvimento de treinamentos, habilidades e percepção para trabalhar atitudes de cada manipulador dentro do processo produtivo de alimentos seguros, podendo adotar programas de gestão de qualidade para toda a UAN.

Segundo Sousa (2017) e Quintino \& Rodolpho (2018), a implantação efetiva das BPF e do sistema APPCC como requisito para o uso de ferramentas reforçam a importância da segurança do alimento como condição fundamental para uma 
evolução da gestão da qualidade na produção de alimentos. Na indústria de alimentos, as BPF são pré-requisitos para se conseguir avançar no quesito garantia da qualidade, bem como para a própria implantação do sistema APPCC.

Tabela 1. Escore Global das condições higienicossanitárias da UAN diariamente. Salvador, Bahia, 2017.

\begin{tabular}{|c|c|c|c|c|c|c|c|}
\hline Blocos & Setores & $1^{\text {a }}$ semana & $2^{a}$ semana & $3^{\mathrm{a}}$ semana & $4^{\text {a }}$ semana & E.O & Classificação \\
\hline 01 & $\begin{array}{l}\text { Recepção de gêneros } \\
\text { alimentícios e área de } \\
\text { circulação }\end{array}$ & $85 \%$ & $76 \%$ & $54 \%$ & $79 \%$ & $73,5 \%$ & Bom \\
\hline 02 & Cocção & $66,4 \%$ & $59,4 \%$ & $69,2 \%$ & $63,6 \%$ & $64,6 \%$ & Regular \\
\hline 03 & $\begin{array}{l}\text { Catação de grãos e } \\
\text { preparo de sucos }\end{array}$ & $68 \%$ & $84 \%$ & $82 \%$ & $90 \%$ & $81 \%$ & Bom \\
\hline 04 & Açougue & $64,8 \%$ & $66,8 \%$ & $64,8 \%$ & $69,8 \%$ & $66,5 \%$ & Regular \\
\hline 05 & $\begin{array}{c}\text { Pré-preparo de } \\
\text { saladas }\end{array}$ & $63 \%$ & $61,8 \%$ & $62,68 \%$ & $62,8 \%$ & $62,5 \%$ & Regular \\
\hline 06 & Sobremesa & $66,8 \%$ & $59,45 \%$ & $64,2 \%$ & $61,6 \%$ & $63 \%$ & Regular \\
\hline 07 & $\begin{array}{l}\text { Higienização de } \\
\text { panelas }\end{array}$ & $52,4 \%$ & $82,4 \%$ & $73,42 \%$ & $61,2 \%$ & $67,3 \%$ & Regular \\
\hline 08 & $\begin{array}{c}\text { Higienização de } \\
\text { bandeja e utensílios }\end{array}$ & $94,2 \%$ & $88 \%$ & $88 \%$ & $89 \%$ & $89,4 \%$ & Bom \\
\hline 09 & Distribuição & $79,4 \%$ & $85 \%$ & $76,8 \%$ & $82,4 \%$ & $80,9 \%$ & Bom \\
\hline 10 & Refeitório & $85 \%$ & $85,5 \%$ & $82,5 \%$ & $87,5 \%$ & $85 \%$ & Bom \\
\hline \multicolumn{6}{|c|}{ Escore Global } & $81,41 \%$ & Bom \\
\hline
\end{tabular}

Legenda: E.O = Escore Obtido. UAN - Unidade de Alimentação e Nutrição.

Fonte: Dados da pesquisa (2017).

Com a aplicação da lista de verificação para avaliação das condições estruturais da Unidade, que eram realizadas de forma semanal, foram incluídas nas observações os espaços físicos do estoque sob refrigeração (antecâmara, câmara dos hortifrutigranjeiro, câmara dos alimentos refrigerados, câmara dos alimentos congelados), estoque seco, estoque de descartáveis e sala da nutrição. Os aspectos relacionados à caracterização da higiene da estrutura física também obtiveram um escore global classificado como bom (71,5\%). Porém, ao observar os setores individualmente, o setor de higienização de panelas obteve pontuação insatisfatória $(48,4 \%)$ para o bloco 15 e crítico $(15 \%)$ para o bloco 5 , enquanto que o setor de prépreparo de saladas também apresentou um escore crítico (30\%) (Tabela 2). Os principais problemas encontrados nestes setores foram decorrentes da falta de higienização dos mesmos, inexistência de equipamentos necessários para pleno desenvolvimento das atividades, problemas decorrentes de estrutura física, falta de fluxo adequado, além da carência de instrumentos como POP de higienização de panelas para auxiliar os manipuladores.

Complementando as boas práticas, a implantação de POP auxiliaria no controle higienicossanitário do processo produtivo de refeições, pois neste instrumento são documentados os procedimentos necessários para assegurar a ausência de perigos que possam comprometer a inocuidade dos alimentos, conforme descrito na RDC n 275/2002 (Brasil, 2002). Para que o POP seja implementado, é fundamental que as responsabilidades pela execução estejam bem estabelecidas. Os serviços de alimentação devem programar POP relacionados aos itens de higienização de instalações, equipamentos e móveis; controle integrado de vetores e pragas urbanas; higienização do reservatório de água e higiene e saúde de manipuladores.

Os estoques refrigerados antecâmara $(52,5 \%)$ e hortifrutigranjeiro $(66,2 \%)$ obtiveram avaliação regular, devido à presença de alimentos impróprios na área, falta de organização do espaço, cruzamento de alimentos pré-preparados com alimentos não higienizados. Resultado semelhante foi encontrado em UAN de escolas públicas do Recôncavo da Bahia, onde o percentual de adequação das condições higienicossanitárias das escolas estudadas foi baixo, variando de 27,2\% a 57,8\%, sendo observado o armazenamento de alimentos juntamente com material de higienização e a rede de frio não adequada ao volume e 
aos diferentes tipos de matérias-primas e ingredientes utilizados no preparo da merenda escolar, não disponibilização do Manual de Boas Práticas e dos Procedimentos Operacionais Padronizados (Almeida, Amor \& Silva, 2018).

Tabela 2. Escore global das condições higienicossanitárias da UAN semanalmente. Salvador, Bahia, 2017.

\begin{tabular}{|c|c|c|c|c|c|c|c|c|}
\hline Bloco & Setores & $\mathbf{1}^{\mathrm{a}}$ semana & $2^{\mathrm{a}}$ semana & $3^{\mathrm{a}}$ semana & $4^{\mathrm{a}}$ semana & $5^{\text {a }}$ semana & E.O & Classificacão \\
\hline 01 & Cocção. & $50 \%$ & $50 \%$ & $75 \%$ & $75 \%$ & $75 \%$ & $65 \%$ & Regular \\
\hline 02 & Açougue. & $75 \%$ & $100 \%$ & $75 \%$ & $100 \%$ & $100 \%$ & $90 \%$ & Bom \\
\hline 03 & $\begin{array}{l}\text { Pré-preparo } \\
\text { de saladas. }\end{array}$ & $75 \%$ & $50 \%$ & $25 \%$ & $0 \%$ & $0 \%$ & $30 \%$ & Crítico \\
\hline 04 & Sobremesa. & $100 \%$ & $75 \%$ & $100 \%$ & $100 \%$ & $100 \%$ & $95 \%$ & Excelente \\
\hline 05 & $\begin{array}{c}\text { Higienização } \\
\text { de panelas. } \\
\text { Higienização }\end{array}$ & $0 \%$ & $0 \%$ & $25 \%$ & $25 \%$ & $25 \%$ & $15 \%$ & Crítico \\
\hline 06 & $\begin{array}{l}\text { de bandejas e } \\
\text { utensílios. }\end{array}$ & $100 \%$ & $100 \%$ & $100 \%$ & $100 \%$ & $100 \%$ & $100 \%$ & Excelente \\
\hline 07 & $\begin{array}{l}\text { Recepção de } \\
\text { gêneros } \\
\text { alimentícios. } \\
\text { Fctoowe }\end{array}$ & $75 \%$ & $87,5 \%$ & $75 \%$ & $75 \%$ & $75 \%$ & $77,5 \%$ & Bom \\
\hline 08 & $\begin{array}{l}\text { refrigerado: } \\
\text { Antecâmara. } \\
\text { Estoque }\end{array}$ & $50 \%$ & $62,5 \%$ & $50 \%$ & $50 \%$ & $50 \%$ & $52,5 \%$ & Regular \\
\hline 09 & $\begin{array}{l}\text { refrigerado: } \\
\text { Hortifruti- } \\
\text { Granjeiro. }\end{array}$ & $77 \%$ & $77 \%$ & $43 \%$ & $67 \%$ & $67 \%$ & $66,2 \%$ & Regular \\
\hline 10 & $\begin{array}{l}\text { Estoque } \\
\text { refrigerado: } \\
\text { Refrigerado. } \\
\text { Estoque }\end{array}$ & $89 \%$ & $83 \%$ & $72 \%$ & $89 \%$ & $89 \%$ & $84,4 \%$ & Bom \\
\hline 11 & $\begin{array}{l}\text { refrigerado: } \\
\text { Congelado. }\end{array}$ & $87,5 \%$ & $87,5 \%$ & $87,5 \%$ & $87,5 \%$ & $87,5 \%$ & $87,5 \%$ & Bom \\
\hline 12 & Estoque seco. & $85 \%$ & $80 \%$ & $75 \%$ & $85 \%$ & $85 \%$ & $82 \%$ & Bom \\
\hline 13 & $\begin{array}{l}\text { Estoque de } \\
\text { descartáveis. }\end{array}$ & $85 \%$ & $85 \%$ & $92 \%$ & $92 \%$ & $92 \%$ & $89,2 \%$ & Bom \\
\hline 14 & $\begin{array}{c}\text { Sala da } \\
\text { nutrição. }\end{array}$ & $83 \%$ & $83 \%$ & $83 \%$ & $83 \%$ & $83 \%$ & $83 \%$ & Bom \\
\hline 15 & $\begin{array}{l}\text { Higienização } \\
\text { de panelas. }\end{array}$ & $53 \%$ & $43 \%$ & $60 \%$ & $43 \%$ & $43 \%$ & $48,4 \%$ & Insatisfatório \\
\hline 16 & $\begin{array}{c}\text { Higienização } \\
\text { de bandejas e } \\
\text { utensílios. }\end{array}$ & $82 \%$ & $82 \%$ & $87 \%$ & $82 \%$ & $82 \%$ & $83 \%$ & Bom \\
\hline \multicolumn{7}{|c|}{ Escore global } & $71,5 \%$ & Bom \\
\hline
\end{tabular}

Legenda: E.O = Escore obtido. UAN = Unidade de Alimentação e Nutrição.

Fonte: Dados da pesquisa (2017).

Ao analisar as duas listas de verificação, percebeu-se que os setores de pré-preparo de salada e higienização de panelas variaram conforme sua classificação, passando de regular para crítico. O que pode ser explicado pelos critérios de avaliação em cada um separadamente, pois durante as observações diárias, questões referentes às condições higienicossanitárias durante o processo produtivo de refeições eram avaliadas, enquanto que nas observações semanais eram atribuídas questões referentes à higiene da estrutura física da Unidade. Estudos evidenciam que é comum o nutricionista assumir a gerência de uma UAN com estrutura física já montada, sendo na maioria das vezes impossível realizar intervenções para melhorias com relação a esta problemática (Sartor \& Alves, 2019; Freitas et al., 2021).

Os setores de preparo de sobremesa e higienização de bandejas e utensílios alcançaram um escore ao nível de serem classificados como excelentes e estes achados foram devido às paredes, teto, portas e janelas estarem sempre higienizados. 
Assim, apesar desses setores não seguirem POP estabelecido, o número de colaboradores alocados em cada um destes setores foi contributivo, pois com um quadro de pessoal maior, fazia com que suas atividades se dessem de forma mais distribuída.

Na presente pesquisa, após a aplicação da lista de verificação, evidenciou-se no geral, que a Unidade não aplicava as BPF de forma satisfatória e consequentemente o APPCC. Todavia, ressalta-se a importância e a obrigatoriedade da implantação destes recursos para a garantia da qualidade higienicossanitária das refeições produzidas (Sousa, 2017; Quintino \& Rodolpho, 2018). Os achados do presente estudo divergem dos de Vitória et al. (2018), pois os mesmos encontraram dados de adequação, sendo que 3,8\% das unidades avaliadas apresentavam baixíssimo risco à saúde e 61,5\% baixo risco sanitário, indicando que a maioria dos locais possuía adequadas condições sanitárias pela presença de BPF.

De forma geral, sabe-se que o APPCC é uma ferramenta preventiva e de gerenciamento, que pode ser usada para proteger os produtos alimentícios contra riscos biológicos, químicos e físicos, sendo então um sistema de base científica e o reconhecimento da existência ou inexistência de formas seguras para o controle de perigos, tendo como objetivo reduzir os riscos de perigos relacionados aos alimentos não sendo um sistema de risco zero (Silva Junior, 2002). O APPCC constitui-se em uma excelente ferramenta de gestão de segurança alimentar, garantindo a eficácia no controle de perigos, sendo aplicável em todo processo de obtenção, elaboração e comercialização de alimentos, desde a aquisição da matéria-prima até o consumo final.

Vale ressaltar então, que o comprometimento da alta direção é indispensável para o início das atividades, essa deve ser informada e motivada para a importância e benefícios que o sistema possa trazer. Nos estudos de Sartor \& Alves (2019) e Freitas et al. (2021) que abordaram perfis de lideranças, enfatizaram a existência de uma sequência lógica, que começa por delegar responsabilidades a um profissional competente e treinado para liderar equipes de trabalho. Esse profissional deverá possuir, além de conhecimento técnico, facilidade de trabalhar em equipe. Assim sendo, dentro de uma UAN, o nutricionista apresenta-se como responsável técnico, com formação que lhe confere capacidade para gerir programas, e para tal, este profissional deve compor o quadro da equipe multidisciplinar a ser formada.

\section{Conclusão}

Considerando o objetivo, faz-se necessário o estabelecimento de um modelo que possibilite a UAN melhorar seu desempenho, que ações e intervenções sejam realizadas com o objetivo de adequar as funções organizacionais ao nível de desenvolvimento que se propõe às unidades de alimentação e nutrição.

No diagnóstico das áreas individualmente observou-se a inadequação dos itens da lista de verificação no qual está relacionado com os resultados insatisfatórios, concluindo, portanto que as boas práticas não são praticadas pelos manipuladores de alimentos do serviço de alimentação e nutrição, sendo necessário o conhecimento e a aplicação das ferramentas de gestão de qualidade como: incluir a realização de procedimentos adequados de Boas Práticas com a implantação de procedimentos de operacionais padronizados para as operações de rotinas, a elaboração de um plano de ação quando da identificação de procedimentos inadequados, com as respectivas ações corretivas.

São necessários que sejam realizadas e aplicadas diariamente estes programas, além de realizar outras pesquisas para que possam contribuir com a melhora da Gestão da Qualidade na produção de alimentos seguros, evitando a possibilidade de causar em eventos adversos à saúde do comensal.

\section{Referências}

Abreu, E. S., Spinelli, M. G. N., \& Zanardi, A. M. P. (2007). Gestão de Unidades de Alimentação e Nutrição: Um modo de fazer. Metha.

Agência Nacional de Vigilância Sanitária - ANVISA. (2002). Resolução RDC $n^{o} 275$, de 21 de outubro de 2002 . Dispõe sobre o regulamento técnico de procedimentos operacionais padronizados aplicados aos estabelecimentos produtores/industrializadores de alimentos e a lista de verificação das boas práticas 
de fabricação em estabelecimentos produtores/ industrializadores de alimentos. Diário Oficial União. https://bvsms.saude.gov.br/bvs/saudelegis/anvisa/2002/anexos/anexo_res0275_21_10_2002_rep.pdf

Agência Nacional de Vigilância Sanitária - ANVISA. (2004). Resolução RDC $n^{\circ} 216$, de 15 de setembro de 2004. Dispõe sobre Regulamento Técnico de Boas Práticas para Serviços de Alimentação. Diário Oficial União. https://bvsms.saude.gov.br/bvs/saudelegis/anvisa/2004/res0216_15_09_2004.html

Almeida, J. S., Amor, A. L. M., \& Silva, I. M. M. (2018). Perfil das merendeiras e inadequação das condições sanitárias e estruturais de escolas de uma cidade do Recôncavo da Bahia - Brasil. REVISTA CEREUS, 10(3), 103-119. http://ojs.unirg.edu.br/index.php/1/article/view/2054

Brasil. (1993). Portaria no 58 de 17 de maio de 1993. Estabelecem Diretrizes e Princípios para a inspeção e Fiscalização Sanitária de Alimentos, Diretrizes e Orientações para o Estabelecimento de Padrões de Identidade e Qualidade de Bens e Serviços na Área de Alimentos - Boas Práticas de Produção e Prestação de Serviços; Regulamento Técnico para Estabelecimento de Padrões de Identidade e Qualidade dos Alimentos. Brasília: Diário Oficial da União. https://bvsms.saude.gov.br/bvs/saudelegis/gm/1993/prt1428_26_11_1993.html

Brasil. (1997). Portaria $n^{\circ} 326$ de 30 de julho 1997. Regulamento técnico sobre as condições higienicossanitarias e de boas práticas de fabricação para estabelecimentos produtores /industrializadores de alimentos. Diário Oficial da União. https://bvsms.saude.gov.br/bvs/saude legis/svs1/1997/prt0326_30_07_1997.html

Brasil. (2010). Decreto $n^{\circ}$ 7.234, de 19 de Julho de 2010. Dispõe sobre o Programa Nacional de Assistência Estudantil - PNAES. Diário Oficial da União, Brasília, DF. Recuperado de: http://www.planalto.gov.br/ccivil_03/_ato2007-2010/2010/decreto/d7234.htm

Cardoso, R. C. V., Góes, J. Â. W., Almeida, R. C. C., Guimarães, A. G., Barreto, D. L., Silva, A. S., Figueiredo, K. V. N. A., Júnior, P. O. V., Silva, E. O. \& Hultner, L. B. (2010). Programa nacional de alimentação escolar: há segurança na produção de alimentos em escolas de Salvador (Bahia)? Rev. Nutr, Campinas, 23(5). https://doi.org/10.1590/S1415-52732010000500010

Chiavenato, I. (2003). Introdução à teoria geral da administração: uma visão abrangente da moderna administração das organizações / - 7. ed. rev. e atual. Rio de Janeiro: Elsevier.

Freitas, J. F., Calazans, D. L. M. S., Medeiros, A. C. Q. \& Alchieri, J. C. (2021). Competências profissionais em saúde: Instrumento de auto avaliação para atuação de Nutricionistas em Alimentação Coletiva. Propriedade Intelectual e a Interdependência entre Ciência, Tecnologia e Inovação. Backup Books Editora. Aracaju. https://www.researchgate.net/profile/Gustavo-Silva-14/publication/351548752_Gestao_de_Patentes_Analise_Tempo ral_e_Quantitativa_dos_D epositos_de_Patentes_nas_Universidades_Federais_Mineiras/links/609cf88c92851cca5989b12f/Gestao-de-Patentes-Analise-Temporal-e-Quantitativa-dosDepositos-de-Patentes-nas-Universidades-Federais-Mineiras.pdf\#page=129

Olmedo, P. V., Stangarlin-Fiori, L., Medeiros, C. O., Tondo, E. C. \& Ferreira, S. M. R. (2017). A profile of foodservices in Curitiba and a critical analysis of the results of sanitary inspections at these establishments. Journal of Food Safety. Vol 38. https://doi.org/10.1111/jfs.12377

Oliveira, J. M. S. (2017). Principais barreiras para a implantação e manutenção do sistema de análise de perigos e pontos críticos de controle (APPCC) em uma micro empresa (MPE). Dissertação (mestrado). Universidade Federal Rural do Rio de Janeiro. https://tede.ufrrj.br/jspui/handle/jspui/4574

Quintino, S. S. \& Rodolpho, D. (2018). Um estudo sobre a importância do APPCC - Análise de Perigos e Pontos Críticos de Controle - na indústria de alimentos. Revista Interface Tecnológica, 15(2), 196-207. https://doi.org/10.31510/infa.v15i2.452

Ribeiro, J. A. (2018). Análise das condições higiênico sanitárias das unidades de alimentação e nutrição das escolas de um município no Vale do Ribeira, SP. Research, Society and Development, 7(8), 01-15. http://dx.doi.org/10.17648/rsd-v7i8.327

Sartor, J. \& Alves, M. K. (2019) Percepção do perfil de liderança do nutricionista gestor em unidades de alimentação e nutrição. Revista Saúde e Desenvolvimento. 7(3). http://dx.doi.org/10.18316/sdh.v7i3.4888

Senac/DN. Guia de Verificação. Boas Práticas e Sistema APPCC. Qualidade e Segurança Alimentar. Projeto APPCC. Mesa. Convênio CNC/CNI/SEBRAE/ ANVISA. 39-54, 2002. Recuperado de: https://silo.tips/download/guia-de-elaboraao-do-plano-appcc

Silva Junior, E. A. (2002). Manual de controle higiênico sanitário em alimentos. 2002. (5a ed.), Varela.

Sobral, F., \& Peci, A. (2008). Administração: teoria e prática no contexto brasileiro. Pearson Prentice Hall.

Sousa, M. D. (2019). Sistemas de Gestão da Qualidade aplicados a Micro e Pequenas Empresas. [Dissertação de mestrado]. https://repositorio.unip.br/wpcontent/uploads/tainacan-items/198/10435/eng_marcosdonizetedesousa.pdf

Souza, C. V. S., Azevedo, P. R. M., \& Seabra, L. M. A. J. (2018). Food safety in Brazilian popular public restaurants: Food handlers' knowledge and practices. Journal of Food Safety. Vol 38. https://doi.org/10.1111/jfs.12512

Stedefeldt, E., Cunha, D. T., Silva Junior, E. A., Silva, S. M. \& Oliveira, A. B. A. (2013). Instrumento de avaliação das Boas Práticas em Unidades de Alimentação e Nutrição Escolar: da concepção à validação. Cien Saude Colet, 18(4), 947-953. https://doi.org/10.1590/S1413-81232013000400006

Susin, V., Pereira, F. B., Gregoletto, M. L. O., \& Cremonese, C. (2017). Food and Nutrition Units conditions and associated aspects. Debate: Society, Science \& Technology, 5(1), 60-68. Recuperado de: https://visaemdebate.incqs.fiocruz.br/index.php/visaemdebate/article/view/820

Teixeira, S., Milet, Z., Carvalho, J. \& Biscontini, T. M. (2000). Administração aplicada a Unidades de Alimentação e Nutrição. Atheneu.

Vitória, A. G., Oliveira, J. S. C., Faria, C. P., \& São José, J. F. B. (2018). Good practices and microbiological quality of food contact surfaces in public school kitchens. Journal of Food Safety. http://dx.doi.org/10.1111/jfs.12486

Vitória, A. G., Oliveira, J. S. C., Pereira, L. C. A., Faria, C. P. \& São José, J. F. B. (2021). Food safety knowledge, attitudes and practices of food handlers: A cross-sectional study in school kitchens in Espírito Santo, Brazil. BMC Public Health 21(1). https://bmcpublichealth .biomedcentral.com/articles/10.1186/s12889-021-10282-1 